\title{
Blindness enhances tactile acuity and haptic 3-D shape discrimination
}

\author{
J. Farley Norman • Ashley N. Bartholomew
}

Published online: 14 June 2011

(C) Psychonomic Society, Inc. 2011

\begin{abstract}
This study compared the sensory and perceptual abilities of the blind and sighted. The 32 participants were required to perform two tasks: tactile grating orientation discrimination (to determine tactile acuity) and haptic threedimensional (3-D) shape discrimination. The results indicated that the blind outperformed their sighted counterparts (individually matched for both age and sex) on both tactile tasks. The improvements in tactile acuity that accompanied blindness occurred for all blind groups (congenital, early, and late). However, the improvements in haptic 3-D shape discrimination only occurred for the early-onset and lateonset blindness groups; the performance of the congenitally blind was no better than that of the sighted controls. The results of the present study demonstrate that blindness does lead to an enhancement of tactile abilities, but they also suggest that early visual experience may play a role in facilitating haptic 3-D shape discrimination.
\end{abstract}

\section{Keywords Shape perception · Blindness $\cdot$ Haptics}

It has long been believed by many that the blind possess heightened sensitivity to nonvisual stimuli. For example, in 1890, Charles Darwin (see also Darwin, 1881, p. 97; Wardrop, 1813) said:

When we direct our whole attention to any one sense, its acuteness is increased; and the continued habit of close attention, as with blind people to that of hearing, and with the blind and deaf to that of touch, appears to improve the sense in question permanently. (p. 361)

J. F. Norman $(\bowtie) \cdot$ A. N. Bartholomew

Department of Psychology, Western Kentucky University,

1906 College Heights Blvd. \#21030,

Bowling Green, KY 42101-1030, USA

e-mail: Farley.Norman@wku.edu
Indeed, a number of modern studies support Darwin's belief that blindness enhances nonvisual perceptual abilities. Wan, Wood, Reutens, and Wilson (2010) found that blind participants exhibited superior performance for auditory pitch discrimination and auditory pitch-timbre categorization when compared to sighted controls. Similarly, Lessard, Paré, Lepore, and Lassonde (1998) found that the blind could localize sounds more accurately than the sighted. This superior performance for the blind is not limited to auditory tasks: Similar enhancements also exist for olfaction. Cuevas, Plaza, Rombaux, De Volder, and Renier (2009) found that their blind participants outperformed sighted controls on three olfactory tasks utilizing 30 "stimulus smells": odor discrimination, free identification, and categorization.

The evidence to date concerning the effects of blindness upon the sense of touch is less clear than for other sensory modalities. Much of this research has been concerned with evaluating the effects of blindness upon tactile acuity: Of eight representative studies, five found superior tactile acuities for the blind when compared to sighted controls (Brown \& Stratton, 1925; Goldreich \& Kanics, 2003; Legge, Madison, Vaughn, Cheong, \& Miller, 2008; Stevens, Foulke, \& Patterson, 1996; Van Boven, Hamilton, Kauffman, Keenan, \& Pascual-Leone, 2000), whereas three found that the tactile acuities of their blind and sighted participants did not differ (Alary et al., 2009; Grant, Thiagarajah, \& Sathian, 2000; Seashore \& Ling, 1918). When faced with such conflicting findings, one would perhaps suppose that these different conclusions occurred because of important differences in methodology, such as the choice of task. However, such differences are difficult to identify. When tactile acuity has been determined by measuring participants' two-point thresholds for the fingertip, the results have been mixed: Some studies (e.g., Brown \& Stratton, 1925) have found that two-point thresholds are significantly lower for the blind, while other studies (e.g., 
Seashore \& Ling, 1918) have found two-point thresholds to be equivalent for blind participants and their sighted controls. When tactile acuity has been measured using grating orientation discrimination, once again the findings have been contradictory: Some studies have found superior discrimination performance for the blind (e.g., Goldreich \& Kanics, 2003; Van Boven et al., 2000), while others (e.g., Alary et al., 2009; Grant et al., 2000) have found no difference in performance between their sighted and blind participants. At the moment, it is unclear whether blind adults truly possess enhanced tactile acuity relative to otherwise similar sighted adults.

When blind and sighted participants are asked to identify two-dimensional (2-D) shapes or pictures (e.g., raised-line drawings), the results are similarly mixed. For example, Heller (1989) found that late-blind participants were much better able to identify raised-line drawings than his sighted controls. Similarly, in a study of congenitally blind and sighted children, D’Angiulli, Kennedy, and Heller (1998) found that the congenitally blind children outperformed their sighted counterparts when both groups actively explored the tactile pictures. However, other studies have found either no difference in performance between blind and sighted participants (Ewart \& Carp, 1963; Picard, Lebaz, Jouffrais, \& Monnier, 2010) or that blind participants' recognition performance was worse than blindfolded sighted participants (Kennedy, 1993, pp. 57-59; Kennedy \& Fox, 1977; Lederman, Klatzky, Chataway, \& Summers, 1990).

The literature concerned with blindness and its effects upon the perception and recognition of tactile pictures is interesting, because many of these pictures are 2-D depictions or projections of common three-dimensional (3-D) objects that many people encounter daily, such as cups, faces, and telephones. However, it is important to keep in mind that these tactile stimuli are pictures, and it has been repeatedly shown that recognizing such tactile pictures is difficult. For example, in Experiment 1 of Lederman et al. (1990; see also D'Angiulli et al., 1998; Heller, 1989; Loomis, Klatzky, \& Lederman, 1991; Wijntjes, van Lienen, Verstijnen, \& Kappers, 2008), their participants' recognition accuracy was only $33.5 \%$. While many previous researchers have studied how blind adults perceive tactile pictures (and Shimizu, Saida, \& Shimura, 1993, have studied the perception of haptic surfaces composed of an array of raised tactor pins), only one previous study (Davidson, Barnes, \& Mullen, 1974) has evaluated blind adults' ability to compare the shapes of solid 3-D objects that they were allowed to actively manipulate. Davidson et al. used as their experimental stimuli a set of 10 solid objects (sculptures), called "feelies," that were created by Gibson (1962, 1963). In Davidson et al.'s task, on any given trial, each blind and sighted participant was required to feel one of the feelies (a "standard" stimulus) for $4 \mathrm{~s}$ and then to feel either three or five "comparison" stimuli for $4 \mathrm{~s}$ each. The participants' task was to indicate which of the comparison stimuli possessed the same 3-D shape as the initial standard stimulus. Davidson et al. found that their blind participants produced significantly fewer errors than did the sighted controls when there were five objects in the comparison arrays.

Does blindness truly affect the sense of touch? If so, does the effect depend upon the particular tactile task that the participants are asked to perform? Perhaps blindness has different effects with regard to tactile acuity and the haptic perception of 3-D object shape. While Darwin (1890, p. 361) appeared to believe that blindness leads to an overall improvement in tactile abilities, Révész (1950, p. 161) referred to a "limited perceptive capacity and apprehension of form by the blind." Indeed, Shimizu et al. (1993, see their Table 2) found that the performance of early-blind participants was significantly worse than that of sighted participants in a haptic identification task. The purpose of the present study was to clarify the abilities of the blind by comparing their tactile acuity and haptic 3-D shape discrimination performance with that of sighted participants matched individually for both age and sex. No previous investigation of blindness and the sense of touch has simultaneously evaluated both tactile acuity and haptic 3-D shape perception for the same group of participants. In addition, no previous study has evaluated blind participants' sensitivity to differences in 3-D shape using standard signal detection measures. A secondary purpose of the present experiment was to remedy this lack of basic information and to assess blind participants' sensitivity to solid shape.

\section{Method}

\section{Participants}

A total of 32 blind and sighted adults participated in the experiment. The participants' ages ranged from 30 to 77 years. The blind participants were either completely blind or could only detect the presence or absence of light (i.e., no pattern vision). As in Wan et al. (2010), our 16 blind participants were classified into three types: (1) the congenitally blind, who were blind at birth or became blind shortly after birth $(N=6)$; (2) the early-onset blind, who became blind before the age of $14(N=5)$; and (3) the late-onset blind, who became blind at or after the age of $14(N=5)$. The blind participants' characteristics are shown in Table 1; all of our blind participants except 1 (Late 2) read Braille. For each of the 16 blind participants, we recruited a sighted control 
Table 1 Characteristics of blind participants

\begin{tabular}{llllll}
\hline ID & Cause of Blindness & Age at Test & Years Blind & Light Perception & Sex \\
\hline Con 1 & Retinopathy of prematurity & 57 & 57 & No & F \\
Con 2 & Anophthalmos/microphthalmos & 43 & 43 & No & F \\
Con 3 & Retinopathy of prematurity & 57 & 57 & Yes & F \\
Con 4 & Retinopathy of prematurity & 60 & 60 & No & M \\
Con 5 & Optic nerve hypoplasia & 68 & 68 & No & F \\
Con 6 & Unknown developmental cause & 51 & 51 & No & M \\
Early 1 & Trauma & 69 & 61 & Yes & M \\
Early 2 & Retinopathy of prematurity & 57 & 51 & No & F \\
Early 3 & Retinitis pigmentosa & 55 & 52 & Yes & F \\
Early 4 & Meningitis & 77 & 74 & No & M \\
Early 5 & Retinopathy of prematurity & 57 & 54 & No & F \\
Late 1 & Congenital glaucoma & 59 & 15 & No & F \\
Late 2 & Aniridia & 65 & 1 & Yes & M \\
Late 3 & Congenital glaucoma & 30 & 16 & No & F \\
Late 4 & Optic nerve hypoplasia & 65 & 40 & No & M \\
Late 5 & Optic nerve hypoplasia & 55 & 13 & Yes & F \\
\hline
\end{tabular}

participant who matched their blind counterpart in terms of both age and sex.

\section{Apparatus}

The random assignment of experimental conditions across trials and the collection of the participants' responses were performed by an Apple MacBook computer.

\section{Experimental stimuli}

In order to evaluate the participants' tactile acuity, we used tactile gratings (JVP Domes; see, e.g., Bleyenheuft \& Thonnard, 2007; Sathian, Zangaladze, Hoffman, \& Grafton, 1997; Van Boven \& Johnson, 1994). JVP Domes (Stoelting, Inc.) are small hemispherical plastic domes that contain linear grooves and ridges of particular widths. We used a set of tactile gratings in which the groove widths ranged from 6 to $0.75 \mathrm{~mm}$ (in particular, $6,5,4,3,2,1.5,1.2,1.0$, and $0.75 \mathrm{~mm}$ ). For the 3-D shape discrimination task, we used a subset of the 12 solid objects (bell peppers) used by Norman, Norman, Clayton, Lianekhammy, and Zielke (2004). These stimulus objects are exact duplicates of natural bell peppers (Capsicum annuum). Rubber molds (the "negatives," made from Evergreen 30 liquid urethane rubber; Smooth-On, Inc.) were created from the original bell peppers; liquid plastic (C-1506 rigid urethane casting compound; Smooth-On, Inc.) was then poured into these molds to create multiple exact "positive" duplicates of the original objects. In order to ensure that the participants' shape discrimination performance was not near ceiling levels (and to leave room for a potential superiority of performance by the blind), only the most difficult pairs of bell peppers from our previous investigation were used: Objects 1 and 3, Objects 1 and 7, Objects 2 and 11, Objects 3 and 7, Objects 3 and 8, and Objects 5 and 12. A photograph of a representative pair of stimulus objects ( 3 and 8$)$ can be seen in Fig. 1.

\section{Procedure}

The participants' tactile acuity was measured using grating orientation discrimination with procedures developed by Van Boven and Johnson (1994; see also Craig \& Kisner, 1998). The advantages of using grating orientation discrimination to measure tactile acuity have been well described by Craig and Johnson (2000). During testing, the tactile gratings were manually applied to the distal fingerpad of each participant's dominant index finger (Bleyenheuft \& Thonnard, 2007, have demonstrated the validity of manual application). The participants' task was to judge (without vision, for the sighted participants) whether the grooves were either aligned parallel or perpendicular to the long axis of their finger. For each groove width, 40 trials were conducted, in which the actual orientation of the grating (parallel vs. perpendicular) was determined at random. Testing generally began at a groove width of $3.0 \mathrm{~mm}$, and continued (with successive blocks of trials using smaller groove widths) until the participants' orientation discrimination performance dropped below threshold levels ( $d^{\prime}$ of 1.35). Once we had found the groove widths that led to discrimination performance that was both above and below each participant's threshold, linear 
Fig. 1 Photograph of a representative pair of objects (Objects 3 and 8 ; these are duplicates of bell peppers) used as stimuli in the haptic 3-D shape discrimination task. These objects were originally created by Norman, Norman, Clayton, Lianekhammy, and Zielke (2004)
3

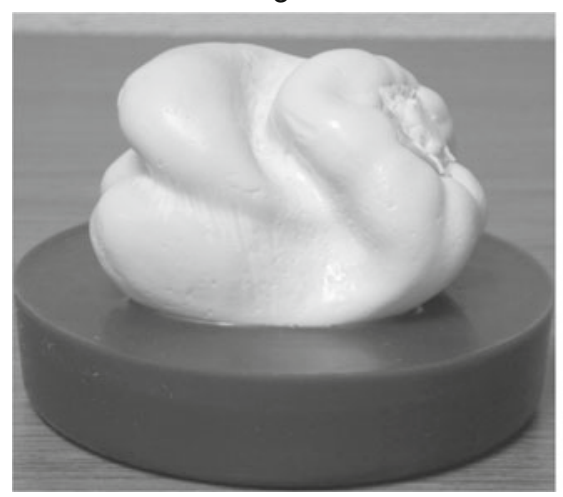

8

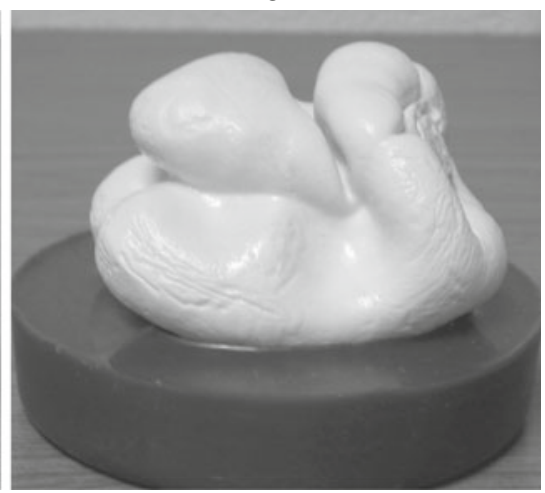

interpolation was used to determine the final threshold estimate (Craig \& Kisner, 1998; Van Boven \& Johnson, 1994). Because aging has been shown to significantly reduce tactile acuity (e.g., Norman et al., 2006, 2011; Stevens, 1992; Vega-Bermudez \& Johnson, 2004; Woodward, 1993), larger groove widths (e.g., 4-6 mm) were sometimes needed in order to determine the tactile acuities of the older participants.

In the 3-D shape discrimination task, the methodology used was essentially identical to the haptic-only conditions of Norman, Clayton, Norman, and Crabtree (2008). On any given trial, two objects were presented sequentially for $3 \mathrm{~s}$ each, separated by an interstimulus interval (ISI) of $3 \mathrm{~s}$. The participants would haptically explore the first object's shape (the sighted participants could not see the objects, because of an occluding curtain) and then similarly explore the second object's shape - they were then required to judge whether the two objects possessed the "same" or "different" 3-D shapes. On every stimulus presentation, the objects were initially placed in completely random 3-D orientations (i.e., every possible 3-D orientation was equally likely). Each participant judged a total of 96 pairs of objects: Half of these were "same" trials, where the two presented objects possessed identical shapes (e.g., Object 1 followed by Object 1), whereas the remaining half of the trials were "different" trials, where the two presented objects possessed different shapes (e.g., Objects 1 and 7). The task was challenging, because even when the same object was presented twice in a row, it was placed in a new, random orientation each time (i.e., does the second object feel "different" because it truly is a different object, or does it feel "different" because it is the same object placed in a completely new, random orientation?).

\section{Results}

The results for the grating orientation task are shown in Fig. 2. The blind participants' thresholds were 30\% lower than the age- and sex-matched sighted controls (thresholds were 2.30 and $1.62 \mathrm{~mm}$ for the sighted and blind participants, respectively; the blind participants could reliably discriminate smaller groove widths than the sighted participants). This difference was statistically significant, according to a matched-sample $t$ test $[t(15)=-3.12, p=.007$, two-tailed]. According to J. Cohen (1988), this is an example of a large effect $(d=0.78)$. An ANOVA conducted upon the blind participants' data indicated that there was no significant difference in tactile acuity across the three types of blindness [congenital vs. early onset vs. late onset: $F(2,13)=0.95$, $p=.41]$. The reason for the lack of a statistical difference between the three blindness groups can be seen in Fig. 2. It is clearly evident that, while the thresholds for the congenitally blind appear to be slightly smaller, on average, than those obtained for the early and late blind, large variability exists within each blindness group relative to the variability between blindness groups. A power analysis revealed that

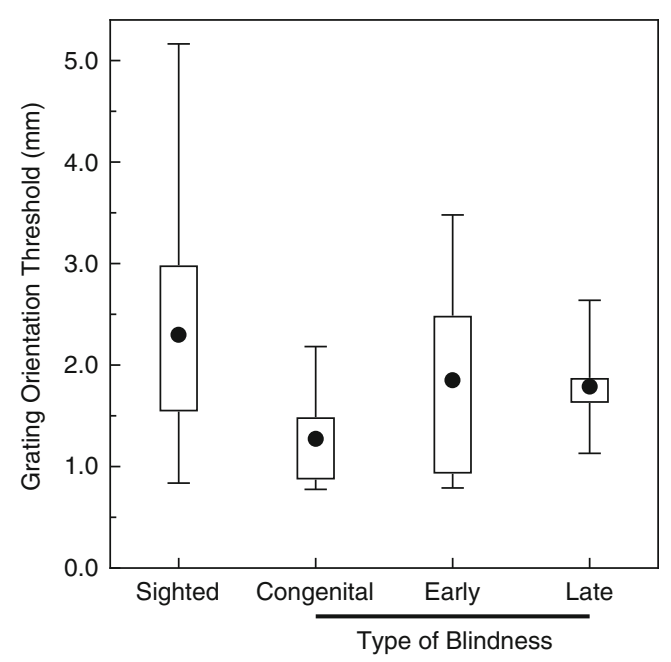

Fig. 2 The grating orientation thresholds are plotted for both the sighted and blind participants; the thresholds for the various blindness groups (congenital, early onset, and late onset) are plotted separately. The results are illustrated with box plots (see, e.g., McGill, Tukey, \& Larsen, 1978). For each group of participants, the top and bottom of the "error bars" indicates the maximum and minimum threshold. The lengths of the boxes indicate the magnitude of the interquartile range (i.e., the distance between the 25 th and 75 th percentiles), while the filled circles indicate the mean threshold for each group 
to have a $90 \%$ chance of detecting a difference this small (i.e., congenital vs. early and late blind), we would need at least 84 blind participants: 42 congenitally blind and 42 early and/or late blind. Our results demonstrate that even if the congenitally blind actually have heightened tactile acuities relative to the early and late blind, this difference is small relative to the individual variability that occurs within any particular blindness group.

The results for haptic 3-D shape discrimination are shown in Fig. 3. The participants' shape discrimination performance is plotted in terms of $d^{\prime}$, which is the measure of perceptual sensitivity used in signal detection theory (see, e.g., Macmillan \& Creelman, 1991). Overall, the blind participants' performance was superior to that of the sighted control participants by approximately $20 \%$ (average $d^{\prime}$ values were 1.908 and 1.592 for the blind and sighted participants, respectively). However, it is important to notice that there were significant differences between the performance of the congenitally blind and the early- and late-onset blindness groups. An ANOVA conducted upon the data shown in Fig. 3 demonstrated that while there was an overall effect of group $[F(3,31)=$ $\left.5.58, p=.004, \eta^{2}=.37\right]$, planned comparisons indicated that only the early and late blind performed significantly better than the sighted participants (both $p$ s <.02). A similar ANOVA conducted upon the blind participants' results revealed significant differences between the various blind groups $\left[F(2,13)=5.91, p=.015, \eta^{2}=.48\right]$. In particular, planned orthogonal comparisons indicated that while the

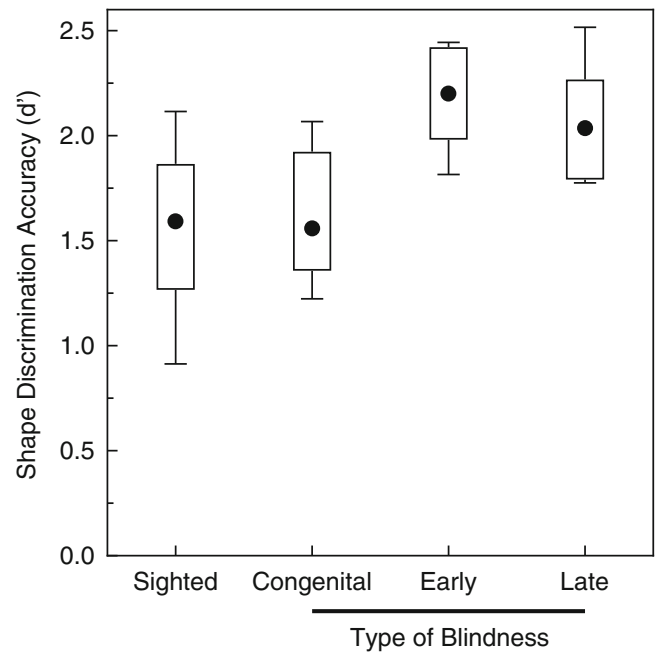

Fig. 3 The shape discrimination accuracies are plotted for the blind and sighted groups of participants. The participants' shape discrimination accuracies are plotted in terms of $d^{\prime}$, the signal detection measure of perceptual sensitivity (see Macmillan \& Creelman, 1991, pp. 141-147). For each group of participants, the top and bottom of the "error bars" indicate the maximum and minimum discrimination performance. The lengths of the boxes indicate the magnitude of the interquartile range (i.e., the distance between the 25th and 75th percentiles), while the filled circles indicate the mean discrimination performance for each group performance of the congenitally blind group was significantly worse (i.e., lower $d^{\prime}$ values) than that exhibited by the early and late blind groups $(p=.005)$, the performance of the earlyand late-onset blindness groups did not differ $(p=.44)$. Finally, it is important to note from an inspection of Fig. 3 that the performance of the congenitally blind was essentially identical to (i.e., not better than) that of the sighted control participants $\left(d^{\prime}\right.$ for sighted participants $=1.59, d^{\prime}$ for the congenitally blind $=1.56$; a power analysis revealed that we would need a total sample of 4,816 participants, 2,408 congenitally blind and 2,408 sighted controls, to have a $90 \%$ chance of detecting a difference as small as that depicted in the left two groups of Fig. 3). It is clear that unlike the early and late blind, the ability of the congenitally blind to haptically discriminate solid shape is not better than that of sighted control participants.

Since we obtained measures of two separate tactile abilities for each participant, it was possible to look for relationships between these measures: Fig. 4 therefore plots each participant's shape discrimination accuracy as a function of their grating orientation threshold. For the sighted participants, there was no relationship between their tactile acuity and their shape judgments $(r=.083, p=.76)$, which is consistent with earlier findings (see, e.g., Norman et al., 2006, 2011). However, for the blind participants, there was a significant correlation between the ability to discriminate tactile grating orientation and the ability to discriminate solid object shape ( $r=.569, p=.021$, two-tailed). The direction of the correlation is interesting, because for our blind participants, reductions in tactile acuity (i.e., larger grating orientation thresholds) were accompanied by improvements in haptic 3-D shape discrimination. It appears that our blind participants

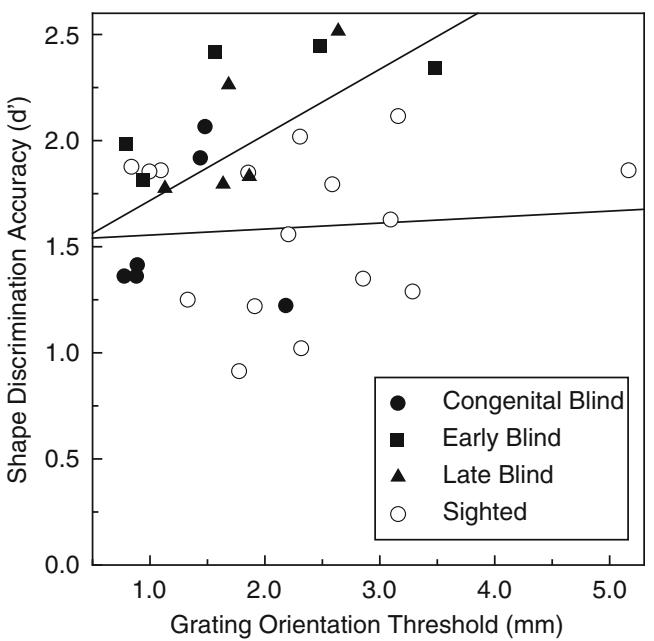

Fig. 4 The shape discrimination accuracies of the individual participants are plotted as a function of their grating orientation thresholds. Results for the blind participants are indicated by the filled symbols, while the open circles indicate results obtained for the sighted participants. The two solid lines represent the best-fitting regression lines for the two groups of participants (blind vs. sighted) 
with poorer tactile acuity were able to compensate for this deficit by developing a heightened ability to discriminate 3-D shape. In this context, it is important to note that an experiment by Klatzky, Loomis, Lederman, Wake, and Fujita (1993) showed that participants could still accurately recognize solid objects while wearing a glove, which would obviously greatly reduce a participant's tactile acuity (see the "realfree" condition results in their Fig. 2): These results of Klatzky et al. clearly demonstrated that excellent haptic object recognition does not necessarily require good tactile acuity.

\section{Discussion}

Our present results documenting an enhancement of tactile acuity for the blind (see Fig. 2) are consistent with a number of previous studies (Brown \& Stratton, 1925; Goldreich \& Kanics, 2003; Legge et al., 2008; Stevens et al., 1996; Van Boven et al., 2000). Quantitatively, our results are quite similar to those of Goldreich and Kanics (2003; e.g., compare our results with their Fig. 4). Our finding of superior tactile acuity in the blind, while similar to the findings of Goldreich and Kanics and Van Boven et al., differs from the results of Grant et al. (2000) and Alary et al. (2009). This difference in outcome is perhaps surprising, because all five of these studies evaluated tactile acuity using a grating orientation discrimination task. However, some possible reasons for the discrepancy exist. In our present study, for every individual blind participant, we recruited a sighted control participant who was exactly matched in terms of both age and sex. If one compares the participants listed in Tables 3 and 4 of Grant et al., it is clear that those authors did not match exactly for age and sex; this was also true in the investigation performed by Alary et al. Given that age does affect tactile acuity (see, e.g., Fig. 10 of Norman et al., 2011; Fig. 5 of Vega-Bermudez \& Johnson, 2004), a failure to match each single pair of participants (i.e., each individual blind participant and their sighted control) would add noise variation to the distributions of the blind and sighted participants' tactile acuities. The presence of "age noise" could be one potential reason for these previous studies' failures to detect enhanced tactile acuity in their blind participants. A second potential reason could be differences in methodology. In our present study, we used blocks of 40 trials for each tactile grating (i.e., for each groove width). In the study by Alary et al., those investigators only presented each tactile grating 20 times (in particular, 160 trials were used to determine thresholds for eight tactilegrating groove widths). We were probably able to estimate our participants' tactile acuity more precisely in the present study because of the increased number of trials (i.e., our dependent measure had higher resolution).
In our experiment, we also found a superiority of the blind in a 3-D shape discrimination task (see Fig. 3). No previous study has evaluated blind participants' ability to discriminate 3-D object shape using signal detection measures, such as $d$ '. This superiority in performance for 3-D shape discrimination was only obtained in the early- and late-onset blindness groups; the haptic shape performance of the congenitally blind was not different from that of the sighted participants (see Fig. 3). This finding is similar to one previously described by Heller (1989), who found that his late-blind participants performed more accurately on a tactile picture (2-D) identification task than did either the congenitally blind or the sighted controls. From the present results and those of Heller, it appears that significant visual experience prior to becoming blind may play a role in the enhancement of haptic shape discrimination or recognition. Other reductions in performance by the congenitally blind have been seen in previous investigations: For example, while the present study and that of Heller found the congenitally blind to be no different than sighted controls, Lederman, Klatzky, Chataway, and Summers (1990; also see Kennedy \& Fox, 1977, Table 5:1) found that their congenitally blind participants performed significantly worse than sighted controls on a tactile picture (2-D) identification task. From the present results and those of Heller, Kennedy and Fox, and Lederman et al., it appears that unlike the early and late blind, congenitally blind adults possess little or no enhancement in haptic shape discrimination or recognition.

To account for patterns of results obtained in their extensive investigations concerning haptics, Klatzky and Lederman (1987) and Lederman et al. (1990) proposed two alternative models of haptic processing: (1) the direct haptic apprehension model and (2) the image-mediation model. In some of their research (e.g., Klatzky, Lederman, \& Metzger, 1985), these authors found excellent haptic performance, in that their participants could accurately recognize common solid objects within a few seconds - these results support the idea that the haptic system is itself responsible for perceiving important properties of solid objects, such as shape (see also Gibson, 1966, pp. 97-135). However, other results obtained within their overall research program (e.g., Lederman et al., 1990) demonstrated that human participants experience difficulty in performing other haptic tasks, such as tactile picture identification (i.e., participants take a long time to make their judgments, which are then often inaccurate). These results suggest that such tasks may involve visual mediation: That is, the participants may convert the tactile input (resulting from the raised contours of the tactile picture) into a visual image, which is then processed and interpreted by the participant's visual system. The involvement of visual mechanisms during the identification of tactile pictures could potentially explain why the congenitally blind 
often perform more poorly than sighted participants during tactile picture identification: Because the congenitally blind have had little or no visual experience, they cannot effectively generate the visual images needed for the visual mediation of tactile input. Some aspects of our present results are consistent with an image-mediation model, but other aspects are inconsistent with this idea. First of all, the fact that our congenitally blind participants performed more poorly with respect to 3-D shape discrimination than did early- and late-blind participants (see Fig. 3) is consistent with an image-mediation model, because unlike the early and late blind, the congenitally blind have had less (or no) experience with visual images. However, in this context, it is important to keep in mind that our congenitally blind participants performed just as accurately (again see Fig. 3) as our sighted participants. Thus, there was no difference between the shape discrimination performance of those participants who have had little or no experience with visual images (the congenitally blind) and that of participants who have had extensive experience with visual images (the sighted controls). In addition, despite the fact that there were significant differences in shape discrimination performance across the various groups of participants (see Fig. 3), all of our participants performed well, with $d^{\prime}$ values averaging above 1.5 for all groups. The fact that our blind and sighted participants performed well, and did so with only 3 -s haptic explorations of the experimental stimulus objects, is consistent with the operation of the direct haptic apprehension model (Klatzky \& Lederman, 1987; Lederman et al., 1990).

As of the present date, many neurophysiological investigations have demonstrated cortical reorganization of function following the onset of blindness, where cortical areas normally responsible for vision become responsive to tactile and haptic input (e.g., Burton, Snyder, Conturo, Akbudak, Ollinger, \& Raichle, 2002; L. G. Cohen et al., 1997; Hyvärinen, Carlson, \& Hyvärinen, 1981; Lanzenberger et al., 2001; Pietrini et al., 2004; Ptito \& Kupers, 2005; Sadato, Okada, Honda, \& Yonekura, 2002; Sadato et al., 1996; Stilla, Hanna, Hu, Mariola, Deshpande, \& Sathian, 2008; Uhl, Franzen, Lindinger, Lang, \& Deecke, 1991; Wanet-Defalque et al., 1988; Weaver \& Stevens, 2007). A number of similar neurophysiological studies have additionally found that more "visual areas" are recruited for tactile and haptic tasks in the late blind than in the congenitally blind (e.g., Büchel, Price, Frackowiak, \& Friston, 1998; Goyal, Hansen, \& Blakemore, 2006). Our present psychophysical finding (as well as the similar findings of Heller, 1989) that the late blind possess enhancements in haptic shape discrimination relative to the congenitally blind may be a reflection of the increased "visual" cortical involvement that occurs for those blind who had significant visual experience prior to becoming blind.

\section{Conclusion}

In general, blind adult participants outperform age- and sexmatched sighted controls on tasks involving the judgment of tactile grating orientation and haptic 3-D shape discrimination. Our results also suggest that early visual experience may play a role in the blindness-related enhancement of solid shape discrimination.

Author Note Among others, we thank the officers and members of the Kentucky chapters of the National Federation of the Blind and the Kentucky Council of the Blind. Their wholehearted support and participation made the research described in this article possible. We also thank John M. Kennedy, Roberta L. Klatzky, and an additional anonymous reviewer for their invaluable help during the preparation of the manuscript.

\section{References}

Alary, F., Duquette, M., Goldstein, R., Chapman, C. E., Voss, P., La Buissonnière-Ariza, V., \& Lepore, F. (2009). Tactile acuity in the blind: A closer look reveals superiority over the sighted in some but not all cutaneous tasks. Neuropsychologia, 47, 2037-2043. doi:10.1016/j.neuropsychologia.2009.03.014

Bleyenheuft, Y., \& Thonnard, J. L. (2007). Tactile spatial resolution measured manually: A validation study. Somatosensory and Motor Research, 24, 111-114.

Brown, M. S., \& Stratton, G. M. (1925). The spatial threshold of touch in blind and in seeing children. Journal of Experimental Psychology, 8, 434-442.

Büchel, C., Price, C., Frackowiak, R. S. J., \& Friston, K. (1998). Different activation patterns in the visual cortex of late and congenitally blind subjects. Brain, 121, 409-419.

Burton, H., Snyder, A. Z., Conturo, T. E., Akbudak, E., Ollinger, J. M., \& Raichle, M. E. (2002). Adaptive changes in early and late blind: A fMRI study of Braille reading. Journal of Neurophysiology, 87, 589-607.

Cohen, J. (1988). Statistical power analysis for the behavioral sciences (2nd ed.). Hillsdale: Erlbaum.

Cohen, L. G., Celnik, P., Pascual-Leone, A., Corwell, B., Faiz, L., Dambrosia, J., . . . Hallett, M. (1997). Functional relevance of cross-modal plasticity in blind humans. Nature, 389, 180-183.

Craig, J. C., \& Johnson, K. O. (2000). The two-point threshold: Not a measure of tactile spatial resolution. Current Directions in Psychological Science, 9, 29-32.

Craig, J. C., \& Kisner, J. M. (1998). Factors affecting tactile spatial acuity. Somatosensory and Motor Research, 15, 29-45.

Cuevas, I., Plaza, P., Rombaux, P., De Volder, A. G., \& Renier, L. (2009). Odour discrimination and identification are improved in early blindness. Neuropsychologia, 47, 3079-3083.

D'Angiulli, A., Kennedy, J. M., \& Heller, M. A. (1998). Blind children recognizing tactile pictures respond like sighted children given guidance in exploration. Scandinavian Journal of Psychology, 39, 187-190.

Darwin, C. (1881). The formation of vegetable mould, through the action of worms, with observations on their habits. London: John Murray.

Darwin, C. (1890). The expression of the emotions in man and animals (2nd ed.). London: John Murray. 
Davidson, P. W., Barnes, J. K., \& Mullen, G. (1974). Differential effects of task memory demand on haptic matching of shape by blind and sighted humans. Neuropsychologia, 12, 395397.

Ewart, A. G., \& Carp, F. M. (1963). Recognition of tactual form by sighted and blind subjects. The American Journal of Psychology, 76, 488-491.

Gibson, J. J. (1962). Observations on active touch. Psychological Review, 69, 477-491. doi:10.1037/h0046962

Gibson, J. J. (1963). The useful dimensions of sensitivity. American Psychologist, 18, 1-15.

Gibson, J. J. (1966). The senses considered as perceptual systems. Boston: Houghton Mifflin.

Goldreich, D., \& Kanics, I. M. (2003). Tactile acuity is enhanced in blindness. Journal of Neuroscience, 23, 3439-3445.

Goyal, M. S., Hansen, P. J., \& Blakemore, C. B. (2006). Tactile perception recruits functionally related visual areas in the late-blind. NeuroReport, 17, 1381-1384. doi:10.1097/01.wnr.0000227990.23046.fe

Grant, A. C., Thiagarajah, M. C., \& Sathian, K. (2000). Tactile perception in blind Braille readers: A psychophysical study of acuity and hyperacuity using gratings and dot patterns. Perception \& Psychophysics, 62, 301-312.

Heller, M. A. (1989). Picture and pattern perception in the sighted and the blind: The advantage of the late blind. Perception, 18, 379389.

Hyvärinen, J., Carlson, S., \& Hyvärinen, L. (1981). Early visual deprivation alters modality of neuronal responses in area 19 of monkey cortex. Neuroscience Letters, 26, 239-243.

Kennedy, J. M. (1993). Drawing and the blind: Pictures to touch. New Haven: Yale University Press.

Kennedy, J. M., \& Fox, N. (1977). Pictures to see and pictures to touch. In D. Perkins \& B. Leondar (Eds.), The arts and cognition (pp. 118-135). Baltimore: Johns Hopkins University Press.

Klatzky, R. L., \& Lederman, S. J. (1987). The intelligent hand. In G. Bower (Ed.), The psychology of learning and motivation: Advances in research and theory (Vol. 21, pp. 121-151). New York: Academic Press.

Klatzky, R. L., Lederman, S. J., \& Metzger, V. A. (1985). Identifying objects by touch: An "expert system". Perception \& Psychophysics, 37, 299-302.

Klatzky, R. L., Loomis, J. M., Lederman, S. J., Wake, H., \& Fujita, N. (1993). Haptic identification of objects and their depictions. Perception \& Psychophysics, 54, 170-178.

Lanzenberger, R., Uhl, F., Windischberger, C., Gartus, A., Streibl, B., Edward, V., . . . Beisteiner, R. (2001). Cross-modal plasticity in congenitally blind subjects. Proceedings of the International Society of Magnetic Resonance in Medicine, 9, 670.

Lederman, S. J., Klatzky, R. L., Chataway, C., \& Summers, C. D. (1990). Visual mediation and the haptic recognition of twodimensional pictures of common objects. Perception \& Psychophysics, 47, 54-64.

Legge, G. E., Madison, C., Vaughn, B. N., Cheong, A. M. Y., \& Miller, J. C. (2008). Retention of high tactile acuity throughout the life span in blindness. Perception \& Psychophysics, 70, 1471-1488. doi:10.3758/PP.70.8.1471

Lessard, N., Paré, M., Lepore, F., \& Lassonde, M. (1998). Early-blind human subjects localize sound sources better than sighted subjects. Nature, 395, 278-280. doi:10.1038/26228

Loomis, J. M., Klatzky, R. L., \& Lederman, S. J. (1991). Similarity of tactual and visual picture recognition with limited field of view. Perception, 20, 167-177.

Macmillan, N. A., \& Creelman, C. D. (1991). Detection theory: A user's guide. New York: Cambridge University Press.

McGill, R., Tukey, J. W., \& Larsen, W. A. (1978). Variations of box plots. The American Statistician, 32, 12-16.
Norman, J. F., Clayton, A. M., Norman, H. F., \& Crabtree, C. E. (2008). Learning to perceive differences in solid shape through vision and touch. Perception, 37, 185-196. doi:10.1068/p5679

Norman, J. F., Crabtree, C. E., Norman, H. F., Moncrief, B. K., Herrmann, M., \& Kapley, N. (2006). Aging and the visual, haptic, and cross-modal perception of natural object shape. Perception, 35, 1383-1395. doi:10.1068/p5504

Norman, J. F., Kappers, A. M. L., Beers, A. M., Scott, A. K., Norman, H. F., \& Koenderink, J. J. (2011). Aging and the haptic perception of 3D surface shape. Attention, Perception, \& Psychophysics, 73, 908-918. doi:10.3758/s13414-010-0053-y

Norman, J. F., Norman, H. F., Clayton, A. M., Lianekhammy, J., \& Zielke, G. (2004). The visual and haptic perception of natural object shape. Perception \& Psychophysics, 66, 342-351.

Picard, D., Lebaz, S., Jouffrais, C., \& Monnier, C. (2010). Haptic recognition of two-dimensional raised-line patterns by earlyblind, late-blind, and blindfolded sighted adults. Perception, 39, 224-235.

Pietrini, P., Furey, M. L., Ricciardi, E., Gobbini, M. I., Wu, W.-H. C., Cohen, L., . . . Haxby, J. V. (2004). Beyond sensory images: Object-based representation in the human ventral pathway. Proceedings of the National Academy of Sciences, 101, 5658-5663.

Ptito, M., \& Kupers, R. (2005). Cross-modal plasticity in early blindness. Journal of Integrative Neuroscience, 4, 479-488.

Révész, G. (1950). Psychology and art of the blind. London: Longman, Green.

Sadato, N., Okada, T., Honda, M., \& Yonekura, Y. (2002). Critical period for cross-modal plasticity in blind humans: A functional MRI study. NeuroImage, 16, 389-400.

Sadato, N., Pascual-Leone, A., Grafman, J., Ibañez, V., Deiber, M.-P., Dold, G., \& Hallett, M. (1996). Activation of the primary visual cortex by Braille reading in blind subjects. Nature, 380, 526-528.

Sathian, K., Zangaladze, A., Hoffman, J. M., \& Grafton, S. T. (1997). Feeling with the mind's eye. NeuroReport, 8, 3877-3881.

Seashore, C. E., \& Ling, T. L. (1918). The comparative sensitiveness of blind and seeing persons. Psychological Monographs, 25 (108), 148-158.

Shimizu, Y., Saida, S., \& Shimura, H. (1993). Tactile pattern recognition by graphic display: Importance of 3-D information for haptic perception of familiar objects. Perception \& Psychophysics, 53, 43-48.

Stevens, J. C. (1992). Aging and the spatial acuity of touch. Journal of Gerontology, 47, P35-P40.

Stevens, J. C., Foulke, E., \& Patterson, M. Q. (1996). Tactile acuity, aging, and Braille reading in long-term blindness. Journal of Experimental Psychology. Applied, 2, 91-106. doi:10.1037/1076-898X.2.2.91

Stilla, R., Hanna, R., Hu, X., Mariola, E., Deshpande, G., \& Sathian, K. (2008). Neural processing underlying tactile microspatial discrimination in the blind: A functional magnetic resonance imaging study. Journal of Vision, 8(10):13, 1-19. doi:10.1167/ 8.10 .13

Uhl, F., Franzen, P., Lindinger, G., Lang, W., \& Deecke, L. (1991). On the functionality of the visually deprived occipital cortex in early blind persons. Neuroscience Letters, 124, 256-259.

Van Boven, R. W., Hamilton, R. H., Kauffman, T., Keenan, J. P., \& Pascual-Leone, A. (2000). Tactile spatial resolution in blind Braille readers. Neurology, 54, 2230-2236.

Van Boven, R. W., \& Johnson, K. O. (1994). The limit of tactile spatial resolution in humans: Grating orientation discrimination at the lip, tongue, and finger. Neurology, 44, 2361-2366.

Vega-Bermudez, F., \& Johnson, K. O. (2004). Fingertip skin conformance accounts, in part, for differences in tactile spatial acuity in young subjects, but not for the decline in spatial acuity with aging. Perception \& Psychophysics, 66, 60-67. 
Wan, C. Y., Wood, A. G., Reutens, D. C., \& Wilson, S. J. (2010). Early but not late-blindness leads to enhanced auditory perception. Neuropsychologia, 48, 344-348. doi:10.1016/j. neuropsychologia.2009.08.016

Wanet-Defalque, M.-C., Veraart, C., De Volder, A., Metz, R., Michel, C., Dooms, G., \& Goffinet, A. (1988). High metabolic activity in the visual cortex of early blind human subjects. Brain Research, 446, 369-373.

Wardrop, J. (1813). History of James Mitchell, a boy born blind and deaf, with an account of the operation performed for the recovery of his sight. London: John Murray.
Weaver, K. E., \& Stevens, A. A. (2007). Attention and sensory interactions within the occipital cortex in the early blind: An fMRI study. Journal of Cognitive Neuroscience, 19, 315-330.

Wijntjes, M. W. A., van Lienen, T., Verstijnen, I. M., \& Kappers, A. M. L. (2008). Look what I have felt: Unidentified haptic line drawings are identified after sketching. Acta Psychologica, 128, 255-263. doi:10.1016/j.actpsy.2008.01.006

Woodward, K. L. (1993). The relationship between skin compliance, age, gender, and tactile discriminative thresholds in humans. Somatosensory and Motor Research, 10, 63-67. 